\title{
Lumbosacral Fusion Using Instrumented Cage Distraction-Fixation in a Dog with Degenerative Lumbosacral Stenosis
}

\author{
Tjarda E. Reints Bok ${ }^{1}$ Lucinda van Stee ${ }^{1}$ Koen Willemsen ${ }^{2}$ Martijn Beukers ${ }^{1}$ Guy C.M. Grinwis ${ }^{3}$ \\ Björn P. Meij ${ }^{1}$
}

${ }^{1}$ Department of Clinical Sciences of Companion Animals, Faculty of Veterinary Medicine, Utrecht University, Utrecht, The Netherlands

2 Department of Orthopaedics, University Medical Centre Utrecht, Utrecht, The Netherlands

${ }^{3}$ Department of Pathobiology, Faculty of Veterinary Medicine, Utrecht University, Utrecht, The Netherlands

\begin{abstract}
Address for correspondence Björn P. Meij, DVM, PhD, Department of Clinical Sciences of Companion Animals, Faculty of Veterinary Medicine, Utrecht University, Yalelaan 108, PO Box 80.154, NL-3508 TD Utrecht, The Netherlands (e-mail: b.p.meij@uu.nl).
\end{abstract}

VCOT Open 2020;3:e77-e83.

\begin{abstract}
Keywords

- distraction-fixation

- degenerative lumbosacral stenosis

- cage

- intervertebral cage

This study aimed to assess the long-term outcome and intervertebral fusion following surgical distraction and stabilization using an intervertebral cage and pedicle screw and rod fixation (PSRF) in a dog with severe degenerative lumbosacral stenosis (DLSS). Degenerative lumbosacral stenosis is a common disorder in large breed dogs and has a multifactorial origin. Surgical treatment by dorsal laminectomy and discectomy results in decompression of neural structures, but when distraction-fixation is applied, the ultimate goal is vertebral fusion. A 4-year-old male neutered Leonberger, presented with DLSS and pre-existent chronic discospondylitis, was treated by dorsal laminectomy, partial discectomy, curettage of the end plates, distraction with an intervertebral spacer (SynCage), and PSRF. At 26 months after surgery, the Helsinki pain score and neurological Griffith score were improved; however, the dog passed away shortly thereafter due to an unrelated disorder. The lumbosacral segment became available for computed tomography $(\mathrm{CT})$, micro-CT and histopathology. On $\mathrm{CT}$, bone volume through the largest hole of the cage was $91.0 \%$ and for compact bone $76.1 \%$. Micro$\mathrm{CT}$ and histopathology revealed vertebral fusion. Distraction-fixation using an intervertebral spacer and PSRF was well-accepted in this dog with severe DLSS, and the dog had a good clinical outcome with long-term follow-up. CT, micro-CT and histopathology showed evidence of vertebral fusion.
\end{abstract}

\section{Introduction}

Degenerative lumbosacral stenosis (DLSS) is a common syndrome affecting mainly large breed dogs. ${ }^{1,2}$ Degenerative lumbosacral stenosis is a multifactorial degenerative disorder characterized by intervertebral disc degeneration/herniation and bony and soft tissue proliferations that contribute to spinal stenosis and cauda equina compression leading to pain, lameness and neurological signs. ${ }^{3}$ Clinical suspicion of DLSS based on low back pain and findings on neurological examination is confirmed by findings on computed tomography (CT) and magnetic resonance imaging (MRI), with MRI giving the most detailed information on bony and soft tissue changes. MRI findings may include intervertebral disc degeneration and received

December 24, 2019

accepted after revision

May 26, 2020
DOI https://doi.org/

10.1055/s-0040-1713824. ISSN 2625-2325.
๑) 2020 Georg Thieme Verlag KG
Stuttgart · New York

License terms

(c) (i) 
herniation, compression of the dural sac and/or nerve roots, focal cauda equina neuritis, foraminal stenosis and bony changes like end plate sclerosis and spondylosis. ${ }^{3-5}$ Conservative medical and surgical treatment have been suggested in veterinary literature, but at this time evidence-based recommendations are lacking. ${ }^{6}$ Surgical treatment consists of dorsal laminectomy, dorsal fenestration (or annulectomy) and nuclear pulpectomy. ${ }^{2,3}$ In case of foraminal stenosis and nerve root compression, the surgery can be expanded to include foraminotomy or distraction with, for instance, pedicle screw and rod fixation (PSRF). ${ }^{7-10}$ Pedicle screw and rod fixation has initially been used to treat pre-existent lumbosacral instability and to prevent further subluxation of the sacrum ${ }^{3,11}$ the safe corridors for the pedicle screws have been described in detail. ${ }^{3,11,12}$ When PSRF is combined with distraction, it also restores the intervertebral disc and foraminal width which is beneficial in case of severe DLSS and foraminal stenosis. ${ }^{9,10}$ Other techniques of fixation at the lumbosacral level include transarticular fixation of the facet joints using pins or transarticular positional screws. ${ }^{13-16}$ Distraction can be further biomechanically supported by the insertion of an intervertebral spacer like a cage which may also promote vertebral fusion which is not achieved by PSRF alone. ${ }^{9,10}$ Vertebral fusion through the cage has been demonstrated in dogs with caudal cervical spondylomyelopathy who were treated with an instrumented intervertebral cage, ${ }^{17}$ but has not been reported previously for the lumbosacral segment.

The aim of the present case report was to describe and evaluate a distraction-stabilization technique using an intervertebral titanium cage and PSRF in a dog with severe DLSS. Outcome was assessed during long-term follow-up, and vertebral fusion was examined using radiography, CT, micro-CT and histopathology.

\section{Case Description}

\section{Animal and Clinical Examination}

A 4-year-old male neutered Leonberger was presented with complaints of difficulty rising, sitting and lying down. On clinical examination, low back pain was evoked when pressure was applied at the lumbosacral junction. On neurologic examination, sciatic and tibial cranial hyporeflexia and pseudo hyperreflexia of the patellar reflex were present, consistent with a lumbosacral lesion localization.

\section{Diagnostic Imaging}

Magnetic resonance imaging (Philips scanner 2013, type Ingenia 1.5T Omega, The Netherlands) showed severe DLSS with signs of chronic pre-existent discospondylitis at the level of L7-S1, characterized by severe intervertebral disc degeneration (Pfirrmann grade $\mathrm{V},{ }^{18}$ ), Hansen type 2 disc protrusion, signal intensity changes of the end plates and vertebral bodies indicating oedema (Modic changes [MC]) type 1, and sclerosis (MC type $3^{19}$ ), L7-S1 foraminal stenosis, central and lateralized compression of neural structures and spondylosis (-Fig. 1). Pre-existing chronic discospondylitis was added as co-existing disease considered likely, based on the severity of the signal intensity changes of the end plates and vertebral bodies.

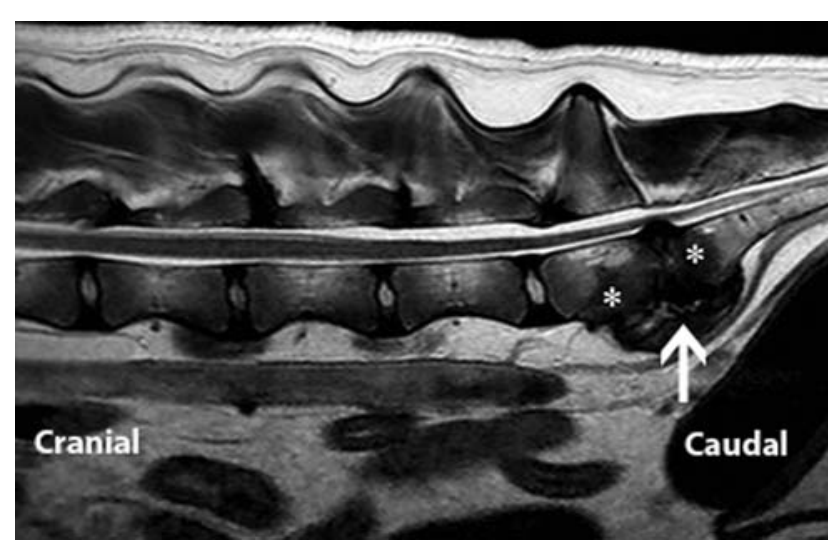

Fig. 1 Sagittal T2-weighted magnetic resonance imaging (T2W MRI) in neutral position. At the level of the white arrow (L7-S1), signal intensity is absent. There is a large amount of hypointense extradural material noted at the ventral aspect of the spinal canal at the level of L7-S1, causing severe compression of the cauda equina at this level. The end plates of L7-S1 are illdefined and along the caudal half of L7 and the cranial half of S1, diffuse hypointense signal is noted $\left(^{*}\right)$. Also, heterogenous T2W hypointense signal is noted ventrally to L7-S1. MRI findings are compatible with chronic preexistent discospondylitis and degenerative lumbosacral stenosis (DLSS).

\section{Surgical Technique}

The dog was initially treated conservatively, including a limited exercise regime and medication, but clinical signs recurred, and surgical treatment was indicated. Medical treatment consisted of a non-steroidal anti-inflammatory drug for dogs given for the patient's weight (Novacam for dogs $1.5 \mathrm{mg} / \mathrm{ml}$, AST Farma, Oudewater, The Netherlands) with a starting dose of $0.2 \mathrm{mg} / \mathrm{kg}$ and continued as $0.1 \mathrm{mg} / \mathrm{kg}$ once daily. In addition, the dog was treated with amoxicillin/clavulanic acid in a dose of $12.5 \mathrm{mg} / \mathrm{kg}$ twice daily (Synulox, Zoetis, Cappele a/d IJssel, the Netherlands). Surgical treatment consisted of dorsal laminectomy at the level of L7-S1 according to the technique described by Danielsson and Sjöström. ${ }^{20}$ Briefly, the dorsal spinous process and caudal half of the dorsal lamina of L7 and the dorsal lamina and dorsal spinous process of S1 were removed leaving the juxtaepiphyseal joints intact. The dorsal spinal process was removed using a Ruskin bone rongeur and the dorsal lamina was removed using a $5 \mathrm{~mm}$ fluted burr (Stryker, Amsterdam, The Netherlands). Bone was collected from the dorsal spinal process and the cancellous bone and cortical bone chips were mixed and saved to be used later as autologous bone graft. Partial discectomy included the dorsal annulus fibrosis and the nucleus pulposus followed by curettage using a 2-mm spoon bone curette. Disc material from the nucleus pulposus cavity was sampled for aerobic and anaerobic microbiological culture. Decompression was followed by instrumented insertion of an intervertebral body spacer. First, two titanium monoaxial pedicle screws (DePuy Synthes, Johnson-Johnson, Oberdorf, Switzerland) were inserted on the right side in S1 and L7 using previously determined safe entry points. ${ }^{12}$ Second, an intervertebral spacer was inserted identical to the one used in patients with cervical spondylomyelopathy. ${ }^{17}$ Distraction with a cervical vertebral body distractor was applied to the pedicle screws which opened the intervertebral disc space. Limited osteostixis of the sclerotic vertebral end plates was performed with a $2.0 \mathrm{~mm}$ drill. A trial cage was inserted with a custom-made 

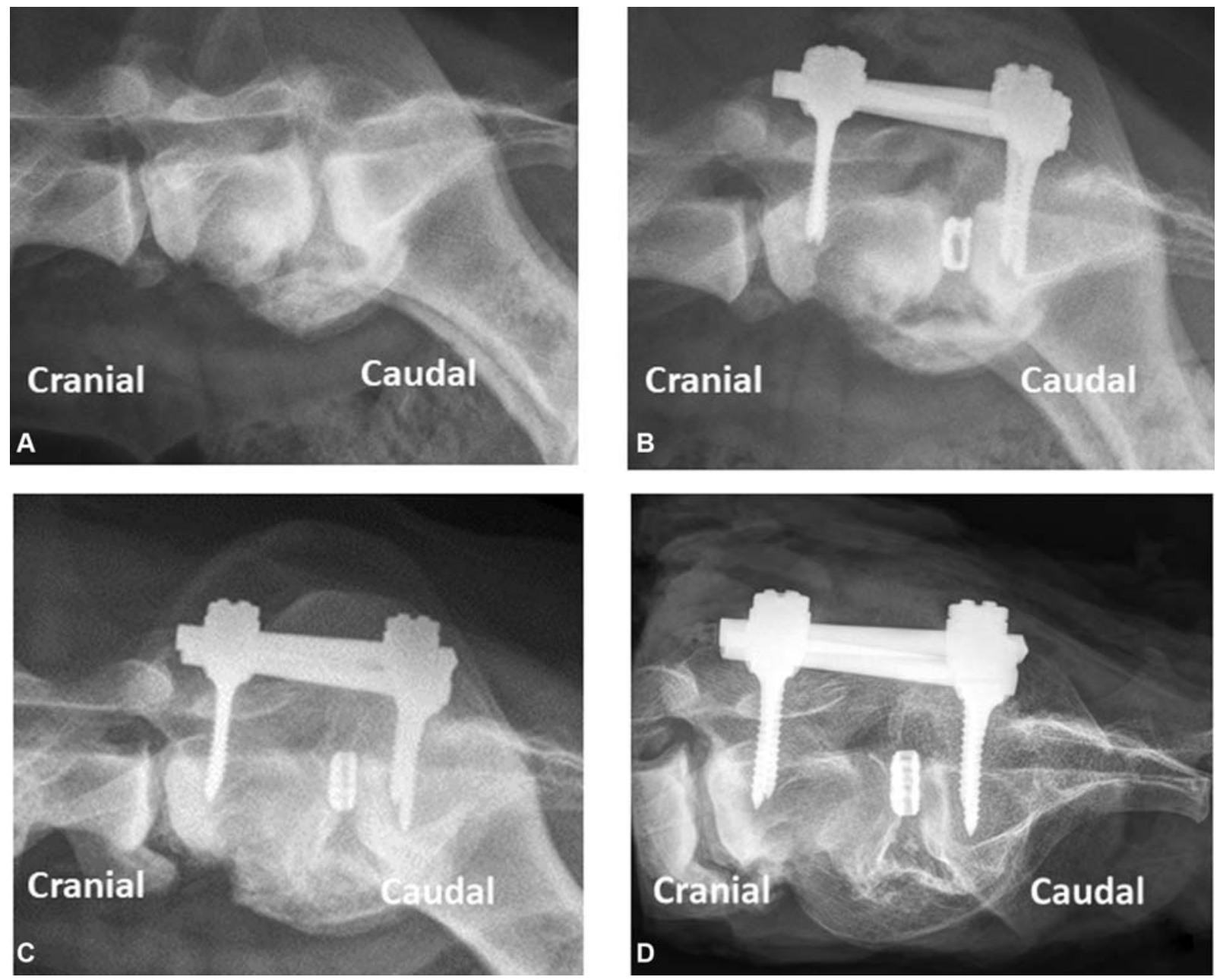

Fig. 2 Left-right lateral radiographs before and after treatment by distraction-fixation using an intervertebral spacer and pedicle screw and rod fixation (PSRF). Preoperative (A), postoperative (B), 12 months postoperative (C) and 26 months (post-mortem) postoperative (D) radiographs. The cage was impacted just ventral to the dorsal surface of the vertebral bodies of L7-S1.

implant holder (DePuy Synthes) and when fit was confirmed, the definitive titanium cage (SynCage-C Short, curved, DePuy Synthes) was inserted and seated just below the dorsal vertebral cortex (- Fig. 2). The cauda equina was carefully deflected laterally, without severe traction, using neurosurgical nontraumatic nerve retractors. Before insertion the cage was filled with the prepared, mixed, cancellous-cortical, autologous bone graft. Third, two monoaxial pedicle screws were inserted on the left side in the same manner as on the right, and L7 and S1 pedicle screws were connected with a $5 \mathrm{~cm}$ long titanium bar (diameter 6-mm) and both bars were firmly tightened to the pedicle screws (-Fig. 2). Finally, a free autogenous fat graft was harvested and placed in the laminectomy defect.

\section{Postoperative Management}

Immediate postoperative care included monitoring of the neurologic status, urinary bladder function, antibiotic therapy and analgesia. The dog went home on carprofen (Carporal, AST Farma, Oudewater, The Netherlands, $2 \mathrm{mg} / \mathrm{kg}$ orally) twice daily for 14 days, tramadol (Tramadol, AST Farma, Oudewater, The Netherlands $5 \mathrm{mg} / \mathrm{kg}$ ) three times daily for 14 days and amoxicillin-clavulanic acid (Synulox, Zoetis, Capelle a.d IJssel, The Netherlands, $13.5 \mathrm{mg} / \mathrm{kg}$ ) twice daily for 10 days, with
6 weeks of leash walks only and slowly increasing the amount of exercise. The amoxicillin-clavulanic acid was stopped when aerobic and anaerobic bacterial culture of the swab returned negative.

\section{Clinical Follow-up}

The dog returned for clinical check-ups at 6 weeks, 6, 7.5, 9 and 12 months after surgery. Low back pain resolved, and at 7.5 months a slight proprioceptive deficit of the left hindlimb was noted, but at the same time a left cranial cruciate ligament rupture was diagnosed. At 1 year after surgery the dog was presented with bilateral cranial cruciate rupture and medial coronoid disease and at 26 months after surgery the dog passed away due to a gastric dilation and volvulus. With the owner's consent, the lumbosacral spine segment was harvested for further examination.

\section{Follow-up Imaging}

Follow-up imaging included radiography (Digital RAD TH, 2008, Philips, The Netherlands) directly postoperatively, and at 6 weeks, and 12 and 26 months after surgery, and CT (Siemens scanner 2014, type Somatom Definition AS 64, The Netherlands) at 9 months after surgery and at 26 months 
(post-mortem). Radiographs were assessed for implant location, structure of the surrounding bone and vertebral fusion. The criteria for the assessment of radiographic fusion were adapted from McAfee and colleagues. Fusion was considered successful if there was evidence of continuous bony bridging between the vertebral bodies and no peri-implant lucency or evidence of implant loosening. Postoperative radiographs and CT imaging were assessed for implant position and adjacent segment pathology, based on disc space narrowing and degenerative changes at the end plates of adjacent vertebral bodies. $^{21}$

The immediate postoperative radiographic view (-Fig. 2B) showed that the lumbosacral spinal unit was fixated in a slight lordotic position correcting the step lesion that was evident on the preoperative radiograph (-Fig. 2A). The lordotic position and the cage positioned in the dorsal half of the vertebral body of L7 and S1 increased the foraminal aperture. In comparison with the postoperative radiograph (-Fig. $\mathbf{2 B}$ ), at 1 year follow-up there was a slight $(4 \mathrm{~mm})$ dorsal displacement of the cage and minimal subsidence in the end plate of L7 (-Fig. 2C). These findings were also evident at CT at 9 months postoperatively (-Fig. 3A), but on all other follow-up imaging the presentation of the implants remained stable ( - Fig. 2D and - Fig. 3B). The cage remained level to the dorsal surface of the vertebral bodies and spondylosis at the level of L7-S1 increased over time (-Fig. 3). On CT at 9 months after surgery, the pedicle screws appeared in the correct corridors of L7 and S1 on the transverse images. There was some degree of adjacent segment pathology visible 9 months after surgery at L6-L7 characterized by disc protrusion.

\section{Vertebral Fusion, Post-mortem Imaging, and Histopathology}

The post-mortem lumbosacral spine segment was fixed and stored in a neutral-buffered $4 \%$ formaldehyde solution for CT, micro-CT and histopathology.

The data of CT and micro-CT were used to measure bone ingrowth through the largest hole in the cage as has been described previously. ${ }^{17}$ In short, the obtained DICOM files were transferred to an image analysis programme (Mimics Medical, v19, Materialise, Leuven, Belgium) and subjected to multi-planar reconstruction to position the largest hole of the cage in line with the normal (craniocaudal) geometrical axis of the cage. A cylinder was fit into the inner volume of the cage ( $5.0 \mathrm{~mm}$ diameter $\times 4.5 \mathrm{~mm}$ length) and isolated using the Boolean subtraction method as the region of interest. MicroCT (Quantum FX, Perkin Elmer, Waltham, Massachusetts, United States) was performed under tube voltage of $90 \mathrm{kV}$, tube current of $180 \mu \mathrm{A}$, scan time of 3 minutes and resolution of $42 \mu \mathrm{m}$. Automated threshold values for compact bone (Hounsfield unit [HU]: 662-1988) and all bone (HU: 266-1988 ) were used to segment the bone in the area of interest. The threshold volume percentage out of the total volume of the region of interest was calculated to determine the amount of bone ingrowth in the largest hole of the cage. On CT there was clear evidence of bone fusion through the largest hole of the cage (-Fig. 3). There was an increase in all bone ingrowth $(85 \%$ to $91 \%$ ) and compact bone ingrowth (55\% to $76 \%$ ), respectively, at 9 months after surgery and in the post-mortem specimen at 26 months after surgery, indicating progression of fusion (-Table 1). Vertebral fusion was also evident on micro-CT at 26 months (-Fig. 3) and measurements were 93\% for all bone and $81 \%$ for compact bone (-Table $\mathbf{1}$ ).
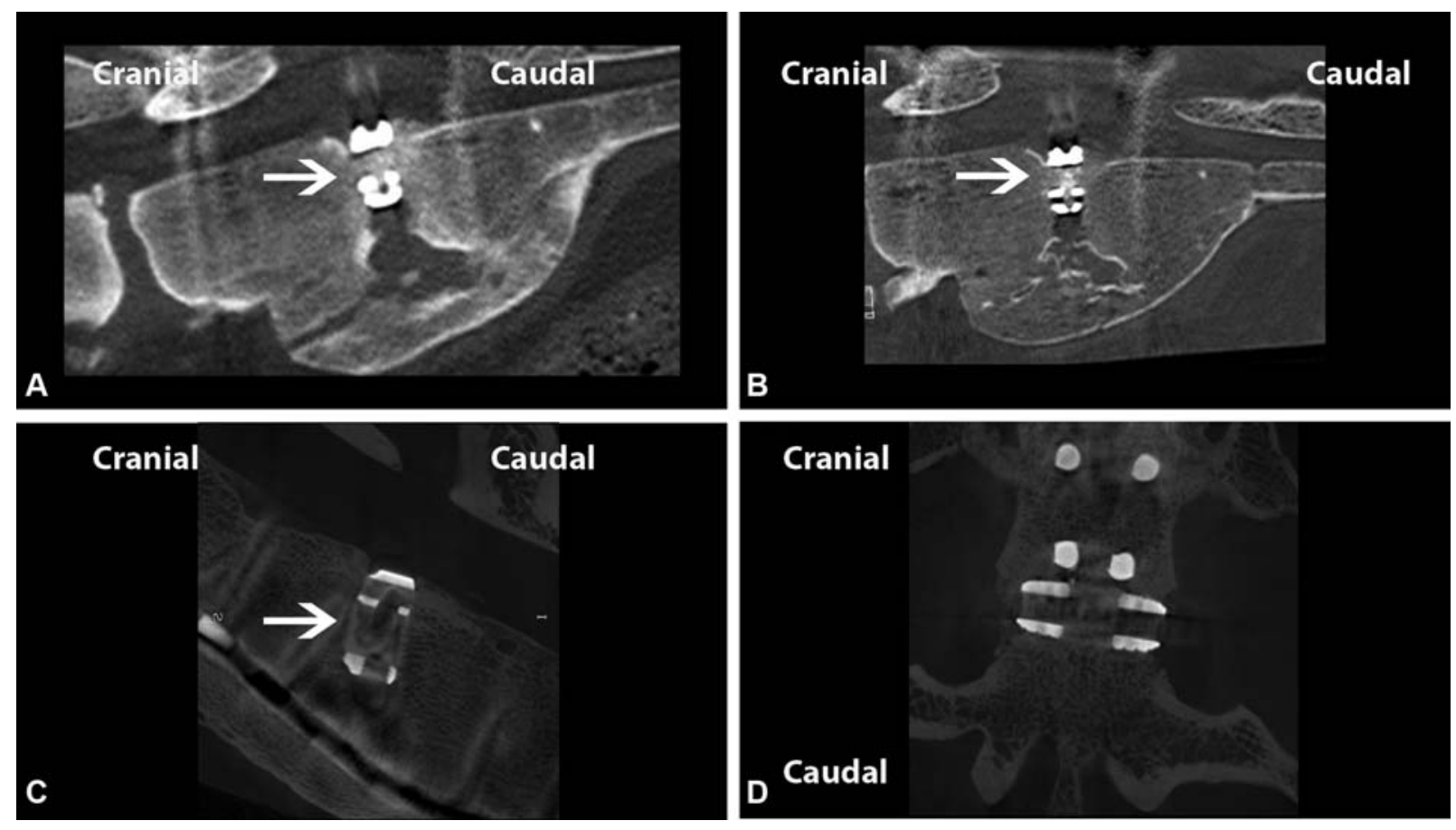

Fig. 3 Sagittal computed tomography (CT) (A, B), sagittal micro-CT (C) and coronal micro-CT (D) reconstruction images of the lumbosacral junction at 9 (A) and 26 (B, C, D) months after treatment by distraction-fixation using an intervertebral spacer and pedicle screw and rod fixation (PSRF). There is prominent bone ingrowth in the largest hole of the cage (arrows). 
Table 1 All bone and compact bone percentages of bone ingrowth through the largest hole of the cage at 9 and 26 months postoperatively

\begin{tabular}{|l|l|l|l|l|l|}
\hline $\begin{array}{l}\text { Follow-up } \\
\text { (months } \\
\text { after surgery) }\end{array}$ & Modality & $\begin{array}{l}\text { \% All bone } \\
\text { threshold: } \\
\text { HU 266-1988 }\end{array}$ & $\begin{array}{l}\text { \% Compact bone } \\
\text { threshold: HU } \\
662-1988\end{array}$ & CT settings & $\begin{array}{l}\text { Titanium } \\
\text { scattering }\end{array}$ \\
\hline 9 & CT & 84.90 & 54.61 & $\begin{array}{l}\text { Slice: } 1 \mathrm{~mm} \\
\text { kV: } 100 \\
\text { MAS: } 341\end{array}$ & $\begin{array}{l}\text { Cage } \\
4 \text { PS } \\
2 \text { rods }\end{array}$ \\
\hline 26 & CT & 91.04 & 76.12 & $\begin{array}{l}\text { Slice: } 1 \mathrm{~mm} \\
\text { kV: } 100 \\
\text { MAS: } 111\end{array}$ & $\begin{array}{l}\text { Cage } \\
4 \text { PS } \\
2 \text { rods }\end{array}$ \\
\hline 26 & Micro CT & 92.79 & 80.75 & $\begin{array}{l}\text { Slice: } 42 \mu \mathrm{m} \\
\text { kV: } 90 \\
\text { MAS: NA }\end{array}$ & $\begin{array}{l}\text { Cage } \\
4 \text { parts of PS }\end{array}$ \\
\hline
\end{tabular}

Abbreviations: CT, computed tomography; HU, Hounsfield unit; kV, Kilovolt; mAs, Milliampere-seconds; PS, pedicle screws.

The lumbosacral spine specimen, including the cage, was processed for histological examination. After dehydration of the sample, using benzoyl peroxide, the material was first embedded in methyl methacrylate before the sample was sawed into histological slides of $\sim 350$ - $\mu$ m using a diamond saw (Leica SP1600, Leica Microsystems, Germany). Histological evaluation of the intervertebral lumbosacral spinal unit showed the presence of well-differentiated trabeculae of lamellar bone and some fibrous tissue within the cage (-Fig. 4). The trabecular bone within the boundaries of the cage appears to be continuous with the bone of both adjacent vertebral bodies. The less organized mixture of bone and fibrous tissue in the central region of the cage probably reflects the mixed cancellous-cortical autologous bone graft that was inserted in the centre of the cage before it was placed in the intervertebral disc.

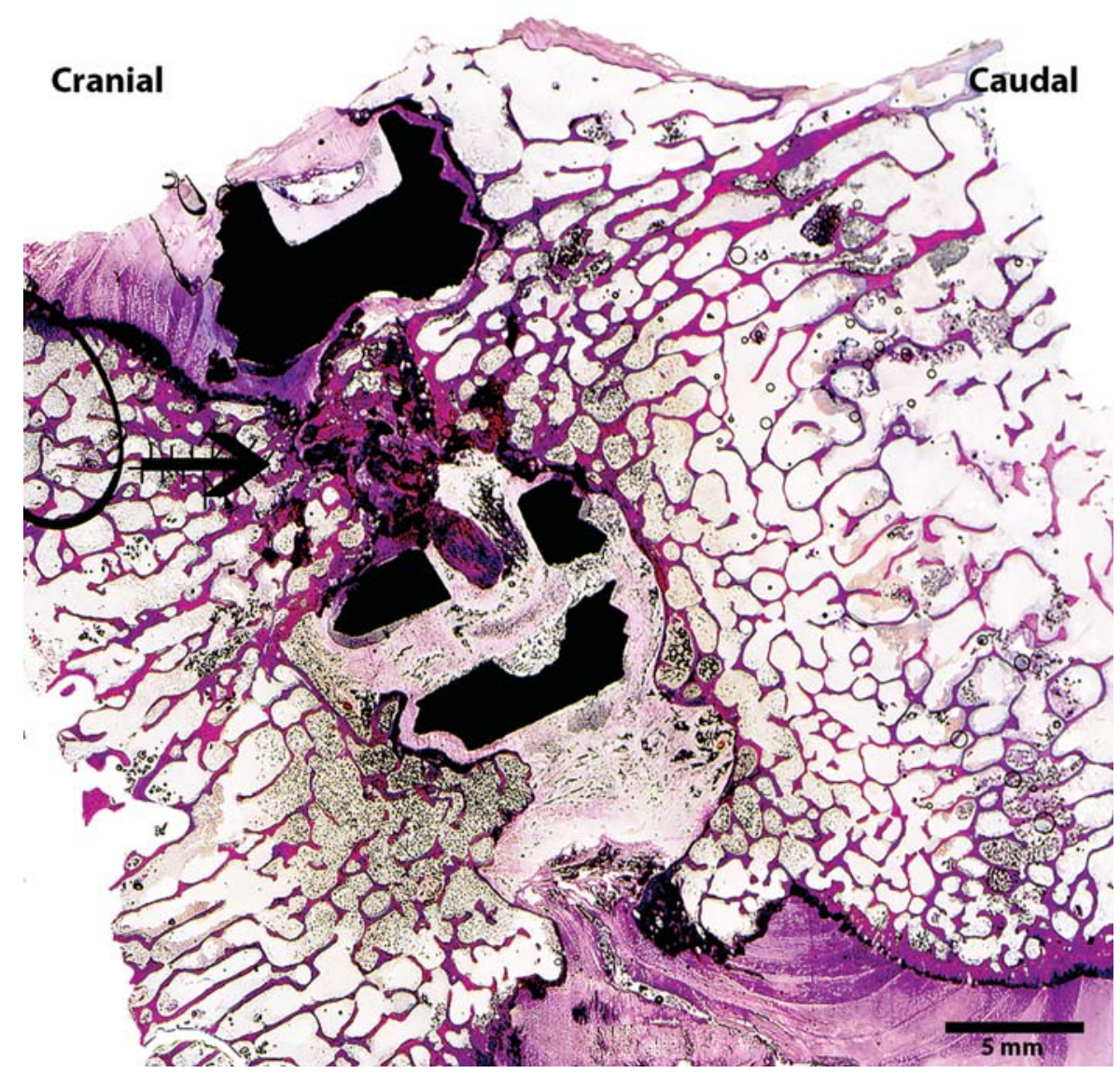

Fig. 4 Histopathology (methylene blue, basic fuchsine staining) of the lumbosacral spinal unit. Bone tissue is stained red-pink, and the titanium cage is stained black. The centre of the cage is largely filled with bone (arrow) and complete fusion of the vertebral body is established. Bar: $5 \mathrm{~mm}$. 


\section{Discussion and Conclusion}

This case study showed that distraction-fixation using an intervertebral cage and PSRF resulted in good clinical outcome for low back pain in a dog with severe DLSS. Clinical follow-up was at 12 months postoperatively, survival was 26 months and the owner's questionnaire reflecting the state of the dog just prior to passing away of the patient. Also, postmortem examination at more than 2 years after surgery revealed evidence of vertebral fusion, both on CT, micro-CT and histological evaluation.

A limitation to this study is that the assessment of bone fusion through the cage is hampered by titanium scattering. However, bone growth was visible on histology and therefore we can assume that at least to some degree the bone growth through the cage has been correctly identified by the measurements on CT and micro-CT. Dorsal migration of the cage and minimal subsidence occurred in the first 9 months postoperatively, and then remained stable. Minimal movement in the first months may have led to delayed bone ingrowth and bone remodelling in the cage, possibly explaining why at 26 months there is still some disorganized bone tissue in some central parts of the cage.

In this patient, it remains unclear why the patient showed proprioceptive deficits of the left hindlimb during clinical follow-up. Dorsal migration of the cage or adjacent segment pathology (ASP) identified at the level of L6-L7 may be responsible for these signs in this patient due to nerve root impingement. After lumbar or lumbosacral fusion in humans ASP was seen in 5.2 to $100 \%$ of patients in several studies; whereas the higher range was seen using only the radiographic criteria. The interval of occurrence of ASP was shorter after instrumented fusion than non-instrumented fusion. However, the actual significance of ASP after fusion remains uncertain. 22

The SynCage was developed for the human cervical spine and is indicated in cervical myelopathy to restore disc width and achieve stabilization through vertebral fusion (DePuy Synthes, Surgical Guide, Johnson and Johnson). Multiple sizes are available for humans; however, for this dog the smallest size $(4.5 \mathrm{~mm})$ was chosen, due to the comparable width of the L7-S1 intervertebral disc in a large breed dog.

It remains to be investigated what the effect is of insertion of the intervertebral cage on the volume of the intervertebral foramen and decompression of the L7 nerves. However, a recent canine cadaveric study in the lumbosacral spine using the same cage showed that intervertebral distraction significantly expanded the L7-S1 intervertebral foramen. ${ }^{23}$

Also, the question arises whether the cage can be used as a stand-alone device or should always be used in an instrumented fashion. In a biomechanical study using the same cage, it was shown that insertion of a stand-alone intervertebral cage (after dorsal laminectomy) restored the vertebral stability, the intervertebral height and the intervertebral foraminal apertures to a state similar to the native (unmodified) spine. Therefore, from a biomechanical standpoint, the use of a stand-alone intervertebral cage seems to be a promising alternative. $^{10}$
In conclusion, this case study showed that there was trabecular bone ingrowth and bone filling of the cage and vertebral spinal fusion achieved by distraction-fixation with an intervertebral spacer (SynCage) and PSRF in a dog with severe DLSS and foraminal stenosis. Whether this is a treatment of first choice in dogs with DLSS needs to be investigated in larger prospective case-studies.

\section{Conflict of Interest \\ None declared.}

\section{References}

1 Oliver JE Jr, Selcer RR, Simpson S. Cauda equina compression from lumbosacral malarticulation and malformation in the dog. J Am Vet Med Assoc 1978;173(02):207-214

2 Suwankong N, Meij BP, Voorhout G, de Boer AH, Hazewinkel HA. Review and retrospective analysis of degenerative lumbosacral stenosis in 156 dogs treated by dorsal laminectomy. Vet Comp Orthop Traumatol 2008;21(03):285-293

3 Meij BP, Bergknut N. Degenerative lumbosacral stenosis in dogs. Vet Clin North Am Small Anim Pract 2010;40(05):9831009

4 Suwankong N, Voorhout G, Hazewinkel HA, Meij BP. Agreement between computed tomography, magnetic resonance imaging, and surgical findings in dogs with degenerative lumbosacral stenosis. J Am Vet Med Assoc 2006;229(12):1924-1929

5 Mayhew PD, Kapatkin AS, Wortman JA, Vite CH. Association of cauda equina compression on magnetic resonance images and clinical signs in dogs with degenerative lumbosacral stenosis. J Am Anim Hosp Assoc 2002;38(06):555-562

6 Jeffery ND, Barker A, Harcourt-Brown T. What progress has been made in the understanding and treatment of degenerative lumbosacral stenosis in dogs during the past 30 years? Vet $\mathrm{J} 2014 ; 201$ (01):9-14

7 Worth A, Meij B, Jeffery N. Canine degenerative lumbosacral stenosis: prevalence, impact and management strategies. Vet Med (Auckl) 2019;10:169-183

8 Gödde T, Steffen F. Surgical treatment of lumbosacral foraminal stenosis using a lateral approach in twenty dogs with degenerative lumbosacral stenosis. Vet Surg 2007;36(07): 705-713

9 Tellegen AR, Willems N, Tryfonidou MA, Meij BP. Pedicle screwrod fixation: a feasible treatment for dogs with severe degenerative lumbosacral stenosis. BMC Vet Res 2015;11:299

10 Teunissen M, van der Veen AJ, Smit TH, Tryfonidou MA, Meij BP. Effect of a titanium cage as a stand-alone device on biomechanical stability in the lumbosacral spine of canine cadavers. Vet J 2017; 220:17-23

11 Meij BP, Suwankong N, Van der Veen AJ, Hazewinkel HA. Biomechanical flexion-extension forces in normal canine lumbosacral cadaver specimens before and after dorsal laminectomydiscectomy and pedicle screw-rod fixation. Vet Surg 2007;36(08): 742-751

12 Smolders LA, Voorhout G, van de Ven R, et al. Pedicle screw-rod fixation of the canine lumbosacral junction. Vet Surg 2012;41 (06):720-732

13 Golini L, Kircher PR, Lewis FI, Steffen F. Transarticular fixation with cortical screws combined with dorsal laminectomy and partial discectomy as surgical treatment of degenerative lumbosacral stenosis in 17 dogs: clinical and computed tomography follow-up. Vet Surg 2014;43(04):405-413

14 Hankin EJ, Jerram RM, Walker AM, King MD, Warman CGA. Transarticular facet screw stabilization and dorsal laminectomy in 26 dogs with degenerative lumbosacral stenosis with instability. Vet Surg 2012;41(05):611-619 
15 Slocum B, Devine T. L7-S1 fixation-fusion for treatment of cauda equina compression in the dog. J Am Vet Med Assoc 1986;188 (01):31-35

16 Bagley RS. Surgical stabilisation of the lumbosacral joint. In: Slatter DH, ed. Textbook of Small Animal Surgery. 3rd edition. Philadelphia: Saunders; 2003:1238-1243

17 Reints Bok TE, Willemsen K, van Rijen MHP, Grinwis GCM, Tryfonidou MA, Meij BP. Instrumented cervical fusion in nine dogs with caudal cervical spondylomyelopathy. Vet Surg 2019;48 (07):1287-1298

18 Bergknut N, Auriemma E, Wijsman S, et al. Evaluation of intervertebral disk degeneration in chondrodystrophic and nonchondrodystrophic dogs by use of Pfirrmann grading of images obtained with low-field magnetic resonance imaging. Am J Vet Res 2011;72 (07):893-898
19 Gendron K, Doherr MG, Gavin P, Lang J. Magnetic resonance imaging characterization of vertebral endplate changes in the dog. Vet Radiol Ultrasound 2012;53(01):50-56

20 Danielsson F, Sjöström L. Surgical treatment of degenerative lumbosacral stenosis in dogs. Vet Surg 1999;28(02):91-98

21 McAfee PC, Boden SD, Brantigan JW, et al. Symposium: a critical discrepancy-a criteria of successful arthrodesis following interbody spinal fusions. Spine 2001;26(03):320-334

22 Park P, Garton HJ, Gala VC, Hoff JT, McGillicuddy JE. Adjacent segment disease after lumbar or lumbosacral fusion: review of the literature. Spine 2004;29(17):1938-1944

23 Smolders LA, Knell SC, Park B, et al. Investigation of the effects of foraminotomy and intervertebral distraction on the volume of the lumbosacral intervertebral foramen: an ex vivo study. Vet Surg 2019;48:658-659 